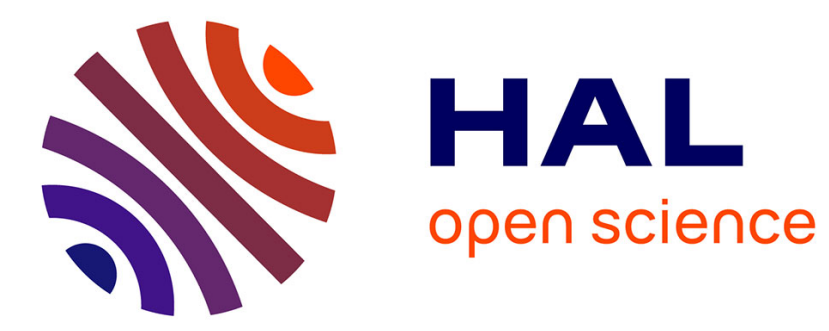

\title{
A Framework for IP and non-IP Multicast Services for Vehicular Networks
}

\author{
Ines Ben Jemaa, Oyunchimeg Shagdar, Thierry Ernst
}

\section{To cite this version:}

Ines Ben Jemaa, Oyunchimeg Shagdar, Thierry Ernst. A Framework for IP and non-IP Multicast Services for Vehicular Networks. NoF 2012 - Third International Conference on the Network of the Future, Nov 2012, Tunis, Tunisia. hal-00762284

\section{HAL Id: hal-00762284 https://hal.inria.fr/hal-00762284}

Submitted on 6 Dec 2012

HAL is a multi-disciplinary open access archive for the deposit and dissemination of scientific research documents, whether they are published or not. The documents may come from teaching and research institutions in France or abroad, or from public or private research centers.
L'archive ouverte pluridisciplinaire HAL, est destinée au dépôt et à la diffusion de documents scientifiques de niveau recherche, publiés ou non, émanant des établissements d'enseignement et de recherche français ou étrangers, des laboratoires publics ou privés. 


\title{
A Framework for IP and non-IP Multicast Services for Vehicular Networks
}

\author{
Ines Ben Jemaa*, Oyunchimeg Shagdar* and Thierry Ernst* \\ *INRIA Rocquencourt, IMARA project-team \\ Domaine de Voluceau BP 105, 78153 Le Chesnay Cedex France \\ Email: \{ines.benjemaa, oyunchimeg.shagdar, thierry.ernst\}@inria.fr
}

\begin{abstract}
Enabling drivers to be connected to the Internet and/or Vehicular Ad-hoc networks, is one of the main challenges of the future networking. This enables drivers to benefit from the existing Internet services as well as emerging ITS applications based on IP or non-IP communications (e.g geonetworking). Many of ITS applications such as fleet management require multicast data delivery. Existing works on this subject tackle mainly the problems of IP multicasting inside the Internet or geocasting in VANETs. This paper presents a new framework that enables Internet-based multicast services on top of VANETs. We introduce a self-configuring multicast addressing scheme based on the geographic locations of the vehicles coupled with a simplified approach that locally manages the group membership to allow packet delivery from the Internet. Moreover, we propose an approach that selects the appropriate network-layer protocol for either geocasting or IP multicasting depending on the vehicles' context and the application requirements. Finally, we present the integration of the designed framework to the ITS reference architecture.
\end{abstract}

\section{INTRODUCTION}

Intelligent Transport Systems (ITS) is expected to largely improve road safety, efficiency, and driving comfort and therefore has received a great attention in both the academics and industry. An important goal of ITS is to provide mobile users (e.g., drivers) to enjoy the existing Internet-based services (e.g., WEB surfing) as well as new types of services dedicated to the highly mobile environment. In order to support such various types of services/applications and over the diverse access media (e.g., DSRC, WiMAX, and 3G), a so-called ITS reference architecture is standardized at ISO and ETSI.

A significant number of ITS applications require multicast communications.For example, a delivery company (center) wants to monitor its trucks on the road, and give a realtime information to the drivers regarding e.g., best routes.This application requires multicast packet delivery from the delivery center to the trucks on the road. Enabling such an application is fraught with challenges, due to the hybrid communications path (the Internet and wireless media) and also the highly mobile nature of the destination nodes. Most of the existing works on multicasting focus only either on infrastructure-less vehicular networks (VANET) [1] [2] or on infrastructure-based networks (e.g., the Internet and WiMAX).

The communication in the Internet is solely based on IP addressing. On the other hand, communications in vehicular networks can utilize geographical addressing techniques (the geographical position of a node is used as the address of the node). The geographical addressing has advantages over the IP addressing, especially for traffic-safety and efficiency applications, where information is often dedicated to the vehicles in a particular geographical area. Much effort [3][4] has been made for efficient geographical addressing and routing; specifications of communications based on geographical addressing is especially conducted in ETSI $^{1}$ and their feasibility has been proven by the R\&D project, namely GeoNet [5], that aggregated the addressing and routing functionalities into a sub-networking layer.

A future challenge is, we believe, to enable multicast communications between the Internet and vehicular networks, especially where different types of addressing techniques are utilized. Figure 1 depicts such a hybrid scenario. In the scenario, a truck detects a hazard on road and broadcasts (i.e., geocast) an alarm message to the immediate surrounding (step $\mathrm{S} 1$ in the figure). The message is also forwarded to a central server, sitting in the Internet, that can process the information and can generate a multicast message destined to the oncoming trucks in a remote area (to recommend an alternative route). In this scenario, the server forwards the message to the upcoming vehicles in the remote area $\mathrm{Y}$ (step S3 and S4 in the figure). Obviously the communications for S2-S4 are based on IP addressing; the communications for S1 and S5 suits more with the geographical addressing techniques.

The above scenario rises the following issues to which we intend to give solutions:

a) Network protocol selection In the scenario, vehicle $A$ sends the message in a geocast fashion (i.e., geobroadcast) to all the surrounding vehicles. The truck $B$ receives the message and transmits it to the central service in the Internet. This observation outlines the necessity of selecting the appropriate destination which can be a geocast group (based on geographical addressing) or a multicast group (based on IP addressing). To deal with this point, we propose a mechanism that dynamically selects the appropriate networking and transport protocol depending on parameters on such as the Internet-connectivity, neighboring information and application type. We particularly consider transmission over UDP and IPv6 in case where the vehicle wants to inform a central server in the Internet

\footnotetext{
${ }^{1}$ ETSI TS 102 636-4-1 V1.1.1Part 4: Geographical addressing and forwarding for point-to-point and point-to-multipoint communications, June 2011
} 


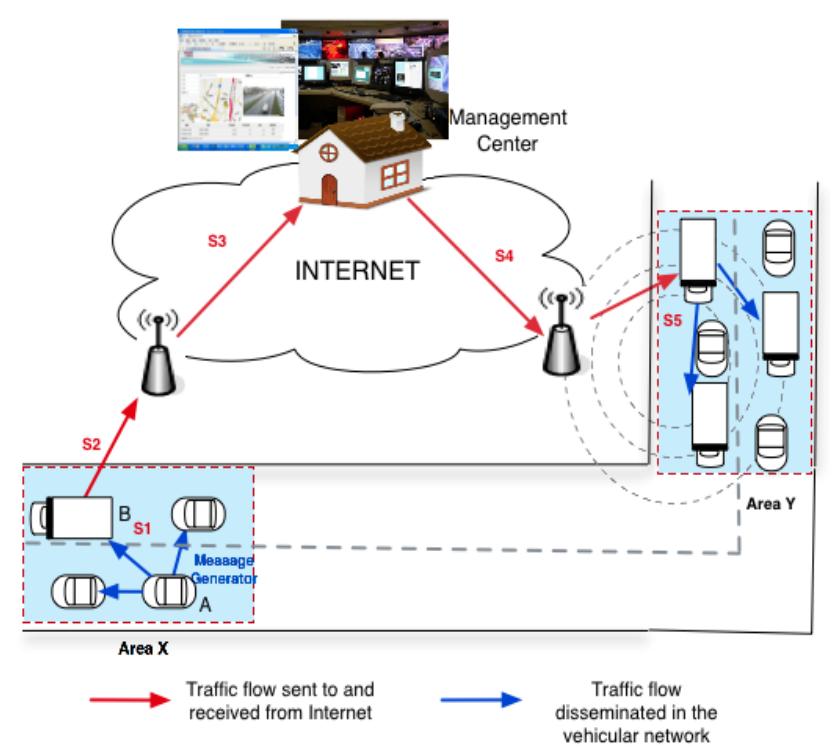

Fig. 1. Multicast hybrid scenario

and transmission using Geonetworking layer coupled with BTP (Basic Transport Protocol) ${ }^{2}$.

b) Group Membership addressing To receive multicast traffic through the Internet or from neighboring vehicles, a group has to be identified by a unique multicast address. Currently, the multicast addresses are assumed to be a priori configured or announced. In ITS scenario, however, we can not bypass the problem of multicast address configuration by assuming that vehicles will keep the same multicast or geocast address when they are moving and changing their geographic location. For this, it is better to give to the vehicles that belong to the same group and are in the same location the responsibility of configuring themselves a common multicast address without requiring negotiation and agreement step. To meet this goal, we propose to deploy a self-configuration of multicast addresses which are correlated to the geographic location of the group members.

c) Multicast data delivery

In ITS scenario, the vehicles form the same group. They are moving and thus changing their geographic location. Changing the geographic location implies also changing the group reachability from the Internet. If the members inform each time they change their location about their membership, this will include routing paths update and maintenance and a signaling overhead. Consequently, the idea of multicast routing based on the informations about the geographic location can be very useful to take into consideration. To meet the above challenges, we propose a simplified approach to deliver packets from the Internet (e.g: central server) to the group of vehicles that are moving in the same area. We basically propose to simplify routing

\footnotetext{
${ }^{2}$ ETSI TS 102 636-5-1 V1.1.1: Sub-part 1: Basic Transport Protocol
}

through the infrastructure by defining a vehicle leader that manages locally the groups in the vehicular network and that is an intermediate node between the infrastructure and the mobile network.

This paper proceeds as follows. The related works are introduced in Section II. In Section III, we describe the requirements for our framework design, and introduces ITS reference architecture.Section IV details the framework design and section $\mathrm{V}$ introduces our development work. Finally we conclude the paper in Section VI.

\section{RELATED WORK}

Much effort has been done to address multicasting in vehicular ad-hoc networks and particularly geographic multicast (geocast) [6]. Although they achieve promising results, these works are focused only on the $\mathrm{V} 2 \mathrm{~V}$ scenario and do not consider Infrastructure-based multicast services. Considering global multicast services, we can not bypass the problems related to multicast addressing and routing.

An early work in [7] allows nodes to auto-configure multicast addresses using the network prefix . The work, however, didn't consider global connectivity to the Internet neither the mobility scenario. Navas and Imielinski in [8] propose solutions to integrate the geographic location to addressing. One solution deals with geo-multicasting which modifies the IP multicast addressing. They also propose to extend Domain Name Service (DNS) with geographical entries. The drawbacks of this scheme is that it requires the modification of the standardized IP multicast addressing.

In [9], a solution that encodes GPS coordinates into the IPv6 multicast address is presented. Nevertheless, this approach remains local and does not scale the whole Internet where the routers are still not geographical addresses aware to the vehicular network.

To route multicast packets, conventional multicast routing scheme on the Internet such as the PIM protocol [10] rely on a multicast distribution tree. This operation is too costly (i.e., large signaling overhead) because it needs to build, update and maintain routing paths along the tree.

[11] introduces a multicast gateway (MGW) to transmit multicast in mixed network; a fixed subnet and a MANET. Unlike our work, this work relies on the infrastructure to deliver multicast packets to the mobile nodes and didn't consider the geographically-scoped data dissemination. The closest approach to our work is, we belive, introduced in [12]. This approach matches the geographic areas to the Access Routers' IP addresses using the extended DNS [8]. In this approach, the packets, once reaching the AR, are disseminated in geobroadcast. But this work doesn't take into consideration the mobility of the multicast group.

\section{ITS REFERENCE ARCHITECTURE AND DESIGN REQUIREMENTS}

As mentioned in Section I, an ITS reference architecture is standardized by ETSI and ISO standardization bodies to support various types of ITS applications over diverse access 
media. Our work has been carried out such that the system design is compliant with the architecture. Moreover, in order to achieve our goals, we define several system requirements that are considered in our framework design. This section introduces the ITS reference architecture and the design requirements.

\section{A. ITS station architecture}

Figure 2 shows the ITS reference architecture. The architecture would be deployed on various types of ITS stations involved in Cooperative ITS communications. This ITS station architecture allows all types of communications: Vehicle-toVehicle (V2V) and Vehicle-to-Roadside (V2R) and Vehicleto-Central (V2C). The major novelties with this architecture is that it introduces a new layer called facilities and two horizontal layers; management and security. The management layer offers cross-layer functions and informations to the whole stack.The facilities layer contains functionality from the OSI application layer, the OSI presentation layer and the OSI session layer. Several data message and components have been standardized. For instance, DENM are alert messages triggered by an application that detects the existence of an event. They carry informations about the event type, the localization, the time of the detection, the relevance area. $C A M$ messages are periodic messages transmitted in single hop mode. They carry information about the current state of the sending station (identifier, position, velocity, etc ...). $L D M$ which is a dynamic map maintains a dynamic network topology of the area around the station.

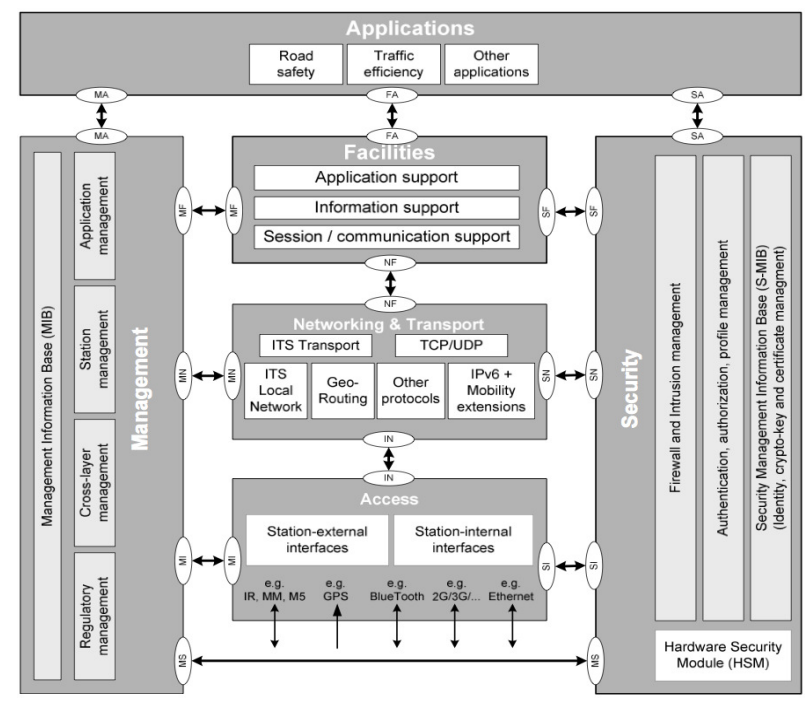

Fig. 2. ITS station reference architecture

\section{B. Design Requirements}

As outlined above, unlike the existing solution for multicast deployment, the framework that we propose aims at providing a dynamic and self-configuring geographic multicast addressing and a simplified approach for packet delivery. To this end, we fix some requirements:
- Scalability: The framework has to offer a global geographic addressing and multicasting delivery service for a large number of groups and members.

- Low complexity and ease of deployment: In a respect of the classic multicast delivery schemes that relies on multicast distribution tree, our approach should offer minimal effort of configuration. Moreover, it has to require minimal changes in case of deployment of new services in the infrastructure or in the vehicular networks.

- Efficiency : This is particularly related to the multicast delivery approach which has to provide low signaling overhead and save the use of the bandwidth especially in the vehicular network.

- Generic for IP and non-IP communication: Our addressing approach has to be generalized for IP and nonIP communication. This is mainly required to host new services that have different requirements. Some services require local multicasting in the direct neighborhood and others require global multicasting through the Internet.

\section{FRAMEWORK PROPOSAL}

The framework that we propose enables two phases: Initialization or bootstrapping phase that includes a geographic multicast auto-configuration process and a group membership building method and a Multicast traffic dissemination phase that includes a network selecting mechanism on the transmission side and a receiver-based multicast delivery in the reception side. In the following sections, we will detail the mechanisms conducted in each phase.

\section{A. Bootstrapping phase}

During this phase, a vehicle that gets into a geographic area performs the geographical-scoped address autoconfiguration (GMAA) operation and the group leader election.

1) Geographic Multicast Address Auto-configuration: GMAA: To bring a solution to the problem of autonomous multicast addressing and configuration for vehicles that are in the same geographic location, we defined a distributed mechanism that allows to the vehicles to configure a common multicast address. The GMAA allows to the vehicle to configure their own address without signaling (i.e, no control message is generated). Furthermore, to support geo-based applications, a vehicle will be able to change the multicast address to which it is subscribed when it changes its location.

We assume that the geographic areas are already partitioned to small areas (for instance, a road can be divided to small segments) and that each vehicle has the same geographic partitioning view. This assumption is reasonable since vehicles are equipped with GPS devices and map-matching capabilities. We also consider that the group identity is already defined implicitly by a profile. The profile includes the vehicle type (i.e: taxi, bus, emergency vehicle...), the motion and the geographic location where the group of vehicle is moving. All the vehicles that have the same profile, have a specific hash function. The hash function $H()$ takes $M$ geographic attributes of the geographic area $p_{i}$ (e.g: for a circular area, 
the geogarphic attributes are the longitude and the latitude of the centre and the radius) where the vehicles are moving and generates a hash value as shown in figure 3 . The hash value is the Group Identifier of the multicast address. The Group Identifier is a sequence of $N$ bits, $h_{i}$ is the bit in position $i$ of the sequence as shown in (1).

$$
H\left(p_{1}, p_{2}, \ldots, p_{M}\right)=\left(h_{1}, h_{2}, \ldots, h_{N}\right) ; h_{i} \in\{0,1\}
$$

An application that runs on the host will subscribe to both a geocast group generated from the process of hashing and a global multicast group generated by concatenating a prefix that has a global scope with the generated Group Identifier. The central server has also the same hash function. Once it receives a message that is relevant to be disseminated to a certain geographic area, it generates the same address. In our work, we used the $F N V$ hash function [13] which is based on multiplication and XOR operation. The reason behind our choice is that the FNV hash function is simple, easy to implement and has a low collision rate which guarantees a higher probability of the uniqueness of the group identifier.

2) Group Membership Management phase: We assume that group members announce themselves through periodical informations exchange that contain their profile, their position and their velocity. One elected vehicle, say leader, within the group plays the role of a local multicast group manager. The leader election process is similar to the approaches such as in [14],[15]. The reason behind using a centralized approach based on group leadership is that the members' mobility will be managed locally. To the central server, only the leader is changing its geographic position and thus its IP address.

\section{B. Multicast Traffic Dissemination phase}

1) The Network Selection: We define a network profile as a set of parameters including the application flows requirements, the available access media interfaces and the availability of an Internet connection. In our work, we basically focus on the ability of the system to select the appropriate networking and transport protocol. As we mentioned before, we consider the case of transmitting geonetworking packets encapsulated in BTP headers and the case of transmitting IP packets encapsulated in UDP/TCP headers. We design a component called Network Selector that intercepts the packets generated by the application and depending on the context decide whether the packets have to be sent using BTP and geonetworking or UDP and IP.

2) The receiver-based multicast delivery: In our approach, the leader notifies periodically its profile. The server performs then the reverse geocoding to conclude the geographic partition where the leader and thus the multicast group members are moving. For each notification it receives, it updates the data structure that stores the meta-characteristics of the geographic area. This is done by filling the leader's entree with the appropriate informations about the leader'profile (IP address, position, velocity...) as shown in figure 3. When the server receives an alerting message for instance that has to be disseminated in a specific geographic area, it builds the multicast address using the GMAA mechanism explained previously. Finally, it encapsulates the multicast packet into a unicast packet using the address of the leader. Once the leader receives the packet, it distributes it to the members of the group that are moving in the same geographic partition using geonetworking capabilities. The time needed to receive the first packet from

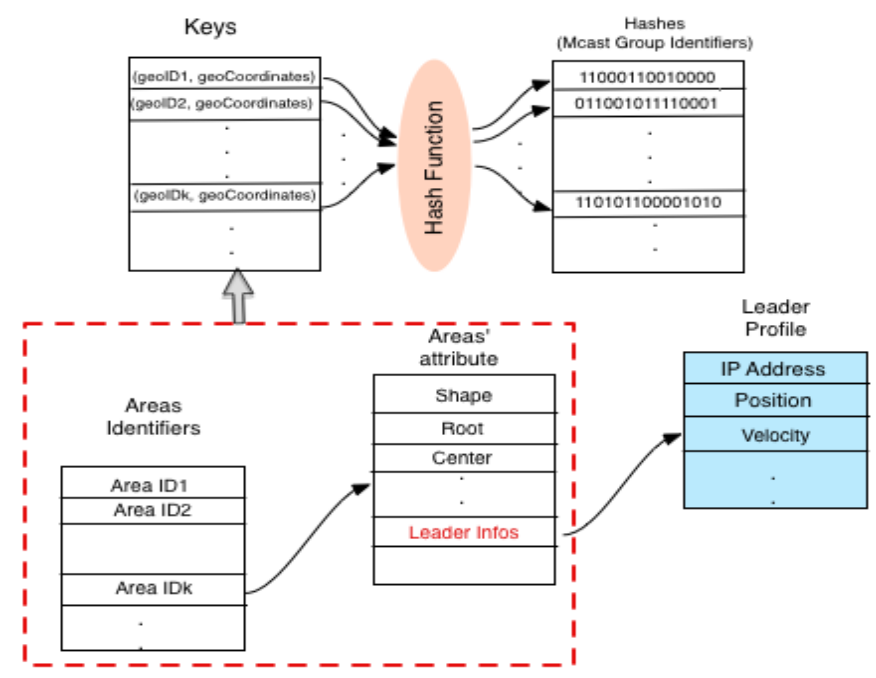

Fig. 3. Hash Function and Area Fetching operation in the server side

the server is given by the following formula:

$$
T_{f} p=T_{f}+T_{h}+T_{t}
$$

$T_{f} p$ is the time needed to receive the first packet in the leader from the server. $T_{f}$ is the time spent by the server in fetching the geographic area attributes (a prototype of the data structure is shown in figure 3 ). $T_{h}$ is the hash process time and $T_{t}$ is the transmission time from the server to the leader. Considering a road segment partition, the time needed to receive the first packet is bounded by the following value

$$
T_{f} p<T_{e}-\frac{L_{s}}{V_{l}}
$$

where $T_{e}$ is the time when the leader enters to the geographic partition, $L_{s}$ is the segment length and $V_{l}$ is the leader's velocity.

\section{FRAMEWORK INTEGRATION DETAILS}

Our framework will be integrated to the ITS architecture described in section III-A. The integration work has been done in a Field Operational Test project; Scoref ${ }^{3}$, which aims at preparing the future deployment of road cooperative systems in Europe. It is compliant to the specification of the ITS reference architecture as explained in section III-A. Our integration is done basically in management and the facilities layer which is implemented in the Knoplerfish ${ }^{4}$ OSGi framework.

\footnotetext{
${ }^{3} \mathrm{http}: / /$ www.scoref.fr/

${ }^{4}$ Knoplerfish http://www.knopflerfish.org/
} 


\section{A. Architecture Design}

Figure 4 outlines the component architecture in the ITS communication architecture. We designed the following components:

- The Mapper is a management layer component. It contains the necessary functions that take as input the geographic attributes of a given area and a hash function (FNV as said before) and generates a unique Mapped Multicast Address per geographic region. It also sends and receives Map matching Request/Reply to and from the LDM.

- The GeoDestination Table this management table stores the geodestination requested from Hazard Application Message DENM for instance (it could be other facilities messages) via GeoArea Request/Reply message exchange.

- The Network Selector Based on predefined rules given by the management plane, the network selector, which is a Facilities component, sends the message through UDP/IPv6 stack or BTP/Geonetworking stack. The rule is related to the previously defined Network Profile. Profile Request/Reply

- The Mapping Table is a kind of dictionary in the management layer that contains the geo-location attributes of a given shape of area and the mapped multicast address.

- The Network Profile Manager collects informations from different layers and depending on these informations attributes the corresponding Communication Profile. The DEMN message for instance, requests the communication profile of the data flow to the Profile Manager and gets a bits array that specifies the transport, network and media access stack where the packet has to be sent.

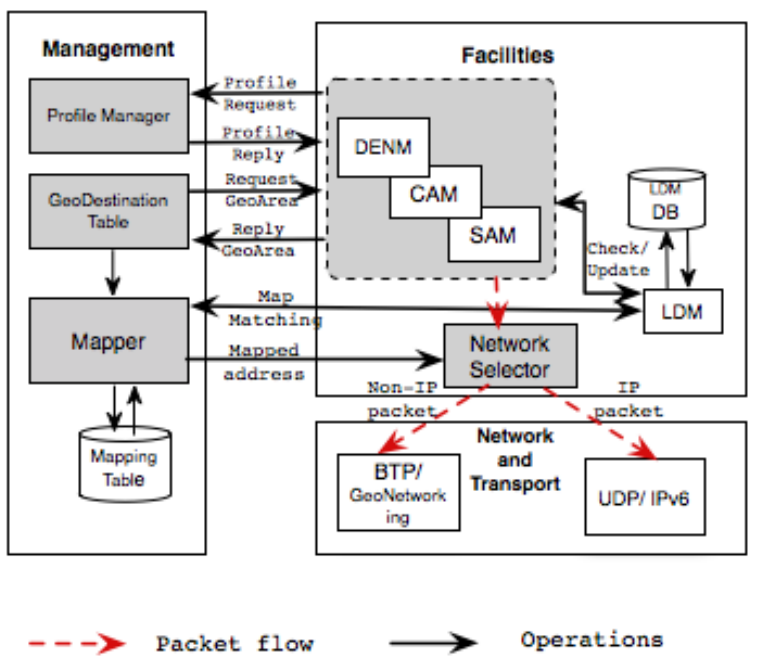

Fig. 4. Architecture Components

\section{B. Framework Operations in the Transmission Side}

Figure 5 shows simplified internal operations performed in both the transmission and the reception side. We design the Network Selector component that allows to select the appropriate protocol in the transmission side. We take the DEMN message as an example. First the DEMN message checks the communication profile via GetCommunicationProfile() function call. The function returns an array of the correspondent communication profile. Once the Network Selector intercepts the message it checks a profile dictionary to get the communication profile meaning (e.g: packets have to be sent over BTP/GeoNetworking and 802.11p access medium). Depending on the returned value, the Network selector set the appropriate dissemination mode (e.g: UNICAST, GEOCAST...) via SetDisseminationMode() and calls the transmissionservices from the appropriate transport protocol.

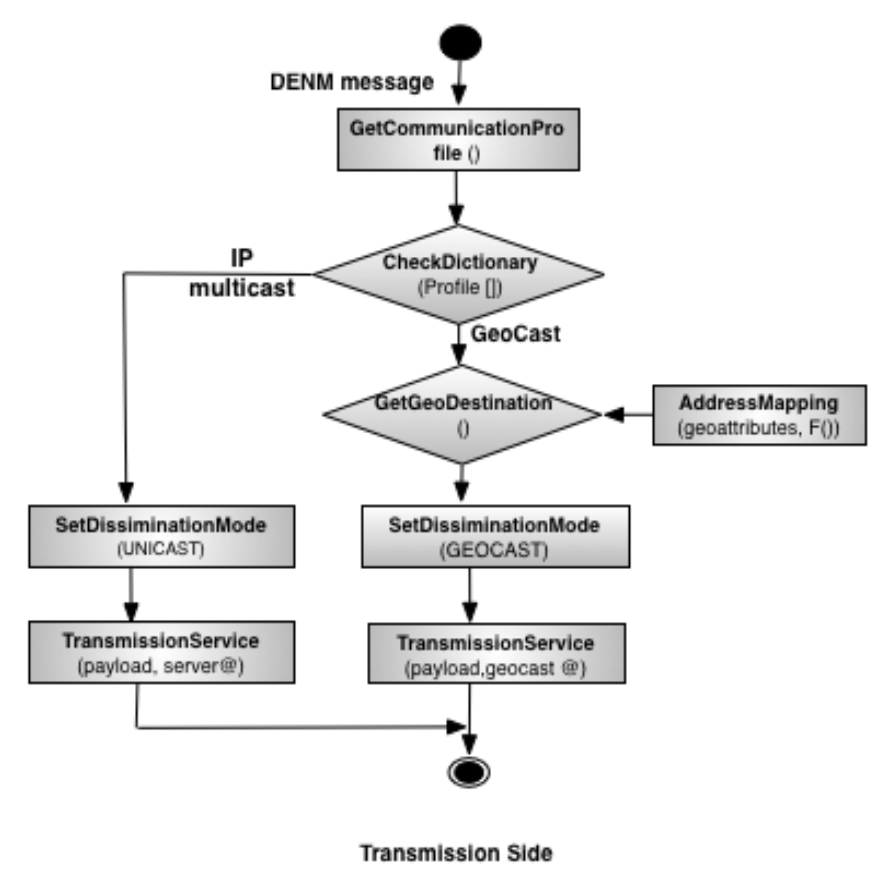

Fig. 5. Activity diagram at packet transmission

\section{Framework Operations in the Reception Side}

Packets are received by the leader and then disseminated to the other vehicles. Figure 6 shows the activity diagram at the leader when it receives a packet from the management server. Before receiving the packet, the Network Selector requests the mapped multicast address using the getMappedAddress(). Once the election process is finished, the leader periodically sends its geographic position to the server using NotifyServer (Pos [],server@) function. Being registered to both BTP port and UDP port, the Network selector receives the payload and then disseminate the message to the group members. 


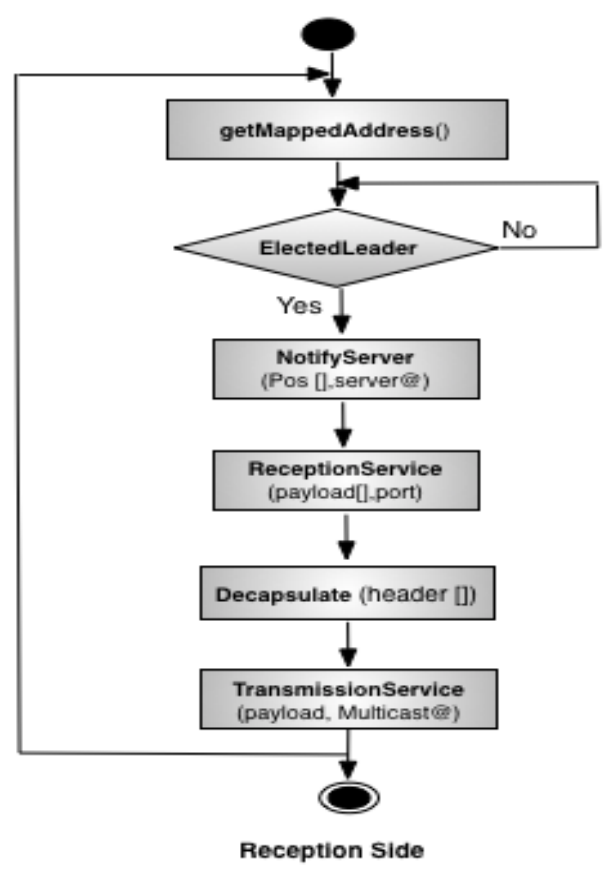

Fig. 6. Activity diagram at packet reception

\section{CONCLUSION AND FUTURE WORK}

In this paper we have introduced a framework that enables deploying multicast services for vehicular networks in Infrastructure-based scenarios. First, we propose a network selecting approach that allows IP and non IP multicast data delivery in the sender side. Then, to meet the challenges of multicast address auto-configuration, we proposed a distributed and efficient geographic multicast auto-addressing mechanism for multicast groups of vehicles. Finally, we introduced a simple multicast data delivery scheme in hybrid networks (i.e Internet to VANETs) from a server to the group of moving vehicles. Currently, we are integrating the framework to the SCOREF project platform. As a future work, we plan to conduct performance evaluation experiments in real operational test field to compare the achieved results with other existing approaches.

\section{REFERENCES}

[1] A. Gordillo Munoz. Multicast over vehicle ad hoc networks. 2009.

[2] Y. Ko and N. Vaidya. Geocasting in mobile ad hoc networks: Locationbased multicast algorithms. WMCSA '99, 1999.

[3] Brad Karp and H. T. Kung. Gpsr: greedy perimeter stateless routing for wireless networks. MobiCom '00, 2000.

[4] SM. Senouci M. Jerbi, R. Meraihi and Y. Ghamri-Doudane. Gytar: improved greedy traffic aware routing protocol for vehicular ad hoc networks in city environments. In Vehicular Ad Hoc Networks'06, 2006.

[5] GeoNet Project, D1.2 Final GeoNet Architecture Design, June 2010.

[6] C. Maihofer. A survey of geocast routing protocols. IEEE Communications Surveys and Tutorials, 2004.

[7] J. Jeong and J. Park. Autoconfiguration technologies for ipv6 multicast service in mobile ad-hoc networks. ICON 2002, 2002.

[8] J. Navas and T. Imielinski. Geocast - geographic addressing and routing. 1997.

[9] Y. Khaled, I. Ben Jemaa, and M. Tsukada. Application of ipv6 multicast to vanet. In ITST'09, 2009.
[10] H. Holbrook B. Fenner, M. Handley and I. Kouvelas. Protocol independent multicast - sparse mode (pim-sm).

[11] W. Ding. Multicast routing in fixed infrastructure and mobile ad hoc wireless networks with a multicast gateway, m.sc. Technical report, 2002.

[12] CJ. Bernardos R. Baldessari and M. Calderon. Geosac - scalable address autoconfiguration for vanet using geographic networking concepts. In PIMRC'08, pages 1-7, 2008.

[13] FNV: http://isthe.com/chongo/tech/comp/fnv/.

[14] P. Caballero-Gil C. Caballero-Gil and J. Molina-Gil. Using groups to reduce communication overhead in vanets. AP2PS, 2010.

[15] Zhigang Wang, Lichuan Liu, MengChu Zhou, and N. Ansari. A positionbased clustering technique for ad hoc intervehicle communication. Trans. Sys. Man Cyber Part C, 2008. 